\title{
Catheter-related Candida bloodstream infection in intensive care unit patients: a subgroup analysis of the China-SCAN study
}

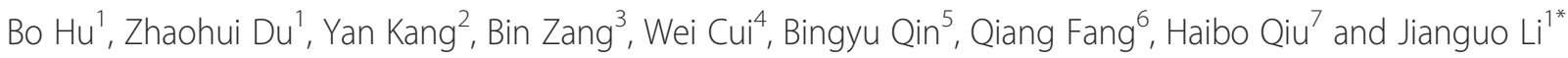

\begin{abstract}
Background: In patients hospitalized in intensive care units (ICU), Candida infections are associated with increased morbidity, mortality and costs. However, previous studies reported confused risk factors for catheter-related Candida bloodstream infection (CRCBSI). The objective was to describe the risk factors, microbiology, management and outcomes of CRCBSI in the China-SCAN population.

Methods: Patients with $\geq 1$ Candida-positive peripheral blood culture were selected from the China-SCAN study. Peripheral and catheter blood samples were collected for Candida isolation. Patients with the same strain of Candida in peripheral and catheter blood samples were considered as being with CRCBSI, while patients with Candida-positive peripheral blood cultures only or different strains were considered as non-CRCBSI. Data were collected from the China-SCAN study.
\end{abstract}

Results: CRCBSI incidence in ICU was $0.03 \%$ (29/96,060), accounting for $9.86 \%$ of all candidemia observed in ICU (29/294). The proportion of CRCBSI due to Candida parapsilosis reached 33.3\%, more than that of Candida albicans (28.6\%). In univariate analyses, older age $(P=0.028)$ and lower body weight $(P=0.037)$ were associated with CRCBSI. Multivariate analysis showed that the sequential organ failure assessment (SOFA) score was independently associated with CRCBSI (odds ratio $(\mathrm{OR})=1.142,95 \%$ confidence interval $=1.049-1.244, P=0.002$ ). Catheter removal and immune enhancement therapy were often used for CRCBSI treatment.

Conclusions: In China, CRCBSI was more likely to occur in old patients with low body weight. SOFA score was independently associated with CRCBSI. Candida parapsilosis accounted for a high proportion of CRCBSI, but the difference from non-CRCBSI was not significant.

Keywords: Catheter related infection, Candidemia, Candida parapsilosis, Candida albicans

\section{Background}

Candida sp. represent the third most common family of pathogens causing bloodstream infections in intensive care units (ICU) patients in the United States [1-3]. The global incidence of candidemia is reported to be 6.7-54 per 1000 ICU patients [4-6]. Untreated candidemia typically results in eye lesions, skin lesions and abscesses, and often lead to multiple organ failure. The mortality rate is 30-61.8\% in Europe and America [5-8]. In addition, candidemia can extend hospital stay by $10-30$ days, and

\footnotetext{
*Correspondence: hobbier1979@163.com

'Department of Intensive Care Unit, Zhongnan Hospital of Wuhan University, Wuhan, Hubei 430071, China

Full list of author information is available at the end of the article
}

increase inpatient hospital costs by about $\$ 40,000$ in the United States [8]. Candidemia requires treatment with an antifungal agent, and removal of the catheter alone is not an adequate therapy for candidemia [9]. The large prospective China Survey of Candidiasis (China-SCAN) study showed that most candidemia in China were caused by non-albicans species (58.2\%), and that first-line antifungal therapy decreased mortality [10].

Catheters are commonly used in ICU patients, and represent an easy entry route for pathogens, including Candida $s p$. In general, patients with candidemia are inserted with catheters, most commonly central venous catheter $(\mathrm{CVC})$, with a placement rate of $80-89 \%$ in Europe and America [11,12]. CVC placement can significantly increase 
the risk of candidemia in hospitalized patients [13], and is an independent risk factor for candidemia in the United States [14]. Candidemia caused by catheter placement is named Candida catheter-related bloodstream infection (CRCBSI). In addition to CVC, studies in Europe and America identified a number of risk factors that are associated with CRCBSI such as surgical trauma, cancer, parenteral nutrition, diabetes mellitus, urinary catheter, age, vancomycin use, and impaired acute physiology and chronic health evaluation (APACHE) score [8,15-19].

The epidemiology of candidemia varies with geography, but is mostly dominated by Candida albicans; however, the proportion of non-albicans candidemia is increasing each year [20], sometimes reaching higher rates than that of albicans candidemia in European countries [21]. In many countries, Candida parapsilosis contributes to $15-20 \%$ of candidemia, and is often associated with CRCBSI [22,23]. Therefore, a better understanding of the CRCBSI epidemiology could lead to better first-line treatments, and to decreased morbidity and mortality.

The China-SCAN study assessed the epidemiology, microbiology, management and outcomes of invasive candidiasis in 67 ICUs across China, and the results were published [10]. The aim of the present study was to assess the risk factors, microbiology, management and outcomes of CRCBSI in the China-SCAN sample. Results might lead to a better identification of patients at high risk of CRCBSI, and to adopt appropriate clinical strategies.

\section{Methods}

\section{Study design and patients}

The methods of the China-SCAN study including inclusion and exclusion criteria were previously published [10]. The present study focused on patients with at least one Candida-positive peripheral blood culture rather than those with positive Candida in histopathological specimen or sterile body cavities fluid specimen culture. Hence, from 306 patients recruited in the CHINA-SCAN study, 294 patients with Candida-positive peripheral blood cultures (290 cases with Candida-positive peripheral blood culture only, and 4 cases with Candida-positive peripheral blood and sterile body cavities fluid specimens) were selected for the present study. Peripheral blood and catheter blood were sampled simultaneously to isolate the Candida strains; the same isolated Candida strain denoted CRCBSI [21,24]. Patients with Candida-positive peripheral blood culture only or with different Candida isolates were considered as non-CRCBSI (NCRCBSI) (Figure 1).

The study was approved by the Ethics Committee of Zhongda Hospital of Southeast University, the lead investigation site. Other participating hospitals accepted the central ethics committee review or conducted a further, independent, ethics review, according to their own institutional policy (20 hospitals). The study complied

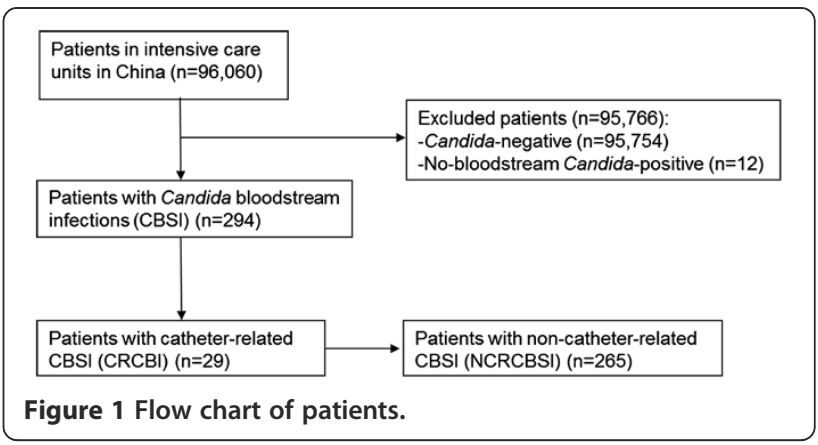

with the Declaration of Helsinki regarding ethical principles of human subjects research and the relevant ethical requirements of the International Conference on Harmonisation/ Good Clinical Practice guidance and national regulations. All patients provided written informed consent. The China-SCAN study is registered with ClinicalTrials. gov (NCTT01253954).

\section{Sample management and strain identification}

All participating centers used the same transportation method. Personnel from each center were trained together to standardize our procedures. Peripheral and catheter blood samples were sampled using strict aseptic methods, and then each sample collected in aerobic and anaerobic blood culture vials $(10 \mathrm{ml}$ each). Interval between peripheral and catheter blood sampling was no more than 5 minutes. Blood samples were immediately sent to the sub-center's laboratory for preliminary screening. After preliminary screening, strains were grown in SDA or PDA plastic tubes at $28-30^{\circ} \mathrm{C}$ and cultured for 2-3 days. After growth, strains were stored at room temperature. Within 1-2 months, strains were transported to the central laboratory. Clinical research assistants at each sub-center were responsible for the regular collection of strains. Strains were shipped to the central laboratory (Research Center for Medical Mycology, Peking University First Hospital, Beijing, China) overnight, at room temperature, by professional courier companies in leak-proof, sealed, unbreakable containers. At the central laboratory, the exact strain and its drug sensibility were determined, as previously described for the China-SCAN study [10,25].

Strains were identified as previously described for the China-SCAN study [10,25]. Briefly, species were identified using chromogenic culture media (CHROMagar, Paris, France) and the API 20C AUX yeast identification kit (bioMérieux SA, Marcy l'Étoile, France). When necessary, large-subunit (26S) ribosomal rRNA gene D1/D2 domain sequencing was performed. Candida haemulonii, Candida pelliculosa, Candida ernobii, Candida norvegensis, Candida metapsilosis and Lodderomyces elongisporus were identified by sequencing. 


\section{Data collection and management}

All data were from the databases of the China-SCAN study. The content and management of the database had been reported in details [10]. Data were collected from case report forms, and analyzed for comparison between CRCBSI and NCRCBSI.

The APACHE II was evaluated within $24 \mathrm{~h}$ of ICU admission. An integrated score from 0 to 71 was calculated. Higher scores indicate more severe disease and higher risk of death [26].

The SOFA score is an ICU scoring system based on organ function or failure rate. The score is based on scores for the respiratory, cardiovascular, hepatic, coagulation, renal and neurological systems [27]. This score was calculated within $24 \mathrm{~h}$ of ICU admission.

Chronic hepatic insufficiency was defined as in APACHE II: 1) biopsy-proven cirrhosis and documented portal hypertension; 2) history of upper gastrointestinal bleeding attributed to portal hypertension; or 3) history of hepatic failure/encephalopathy/coma.

\section{Statistical analysis}

Statistical analysis was conducted using SAS 9.1 (SAS Institute, Cary, NC, USA). Continuous variables are presented as means \pm standard deviation (SD) (normally distributed), or median (Q1, Q3) (non-normally distributed). Categorical variables are presented as frequencies and percentiles. Independent samples $t$-tests and Wilcoxon rank sum tests were used for continuous variables, as appropriate. Chi-square tests and Fisher's tests were used for categorical data, as appropriate. Variables with $P$-values $<0.20$ in univariate analyses were entered into a multivariate logistic regression analysis to identify factors that were independently associated with CRCBSI. $P$-values $<0.05$ were considered to be statistically significant.

\section{Results and discussion}

Incidence and patient characteristics

Among the 294 identified patients, there were 265 cases of NCRCBSI and 29 cases of CRCBSI. Patients with CRCBSI represented $9.86 \%$ of all candidemia patients. Based on the 96,060 ICU patients in the CHINA-SCAN study [10], CRCBSI incidence was $0.03 \%$ of ICU patients (29/96,060) (Figure 1).

The baseline characteristics of patients are compared in Table 1. Results from univariate analyses suggest that CRCBSI was associated with age (69.4 $\pm 19.1 v s$. $60.7 \pm 20.2$ years, $P=0.028$ ) and with body weight $(58.0 \pm 5.2$ vs. $63.2 \pm 11.0 \mathrm{~kg}, P=0.037)$. SOFA score at candidemia diagnosis $(P=0.15)$, solid tumors $(P=0.18)$ and chronic hepatic insufficiency $(P=0.06)$ were also included in the multivariate analysis.

\section{Catheter indwelling between the CRCBSI and NCRCBSI groups}

Among CRCBSI patients, 26 were inserted with CVC, and the remaining three had peripheral arterial catheters. Among the NCRCBSI patients, 217 were inserted with CVC. There was no difference in the rate of CVC indwelling between CRCBSI and NCRCBSI patients $(P=0.438)$. CVC puncture was placed in the jugular, subclavian, or femoral vein, without difference between the groups (all $P>0.05$ ). Indwelling time of the last catheter before candidemia diagnosis was not different between the groups (all $P>0.05$ ) (Table 2).

\section{Catheter removal between the CRCBSI and NCRCBSI groups}

There was no difference between the two groups in the number of CVC removal from each position within two weeks before candidemia diagnosis (all $P>0.05$ ). Significantly more catheters were removed in the CRCBSI group after diagnosis $(82.8$ vs. $60.0 \%, P=0.016)$ (Table 2$)$.

\section{Microbiology}

Because of the individual hospital policy and suboptimal storage or handling of isolates, not all isolates from other hospitals were sent to the central laboratory for review and identification, and a total of 237 isolates (21 from CRCBSI patients, and 216 from NCRCBSI patients) were identified in the central laboratory (Table 3). The proportion of Candida parapsilosis (33.3\%) was higher than that of Candida albicans (28.6\%) in the CRCBSI group, while the proportion of Candida albicans (40.3\%) was the highest in the NCRCBSI group. However, there was no difference in the distribution of Candida strains between the two groups $(P=0.352)$. The results derived from the different hospitals were consistent with those obtained in the central laboratory. Additional file 1: Table S1 presents the strains distribution across study centers in China.

\section{Multivariate analysis}

Multivariate analysis showed that the SOFA score at candidemia diagnosis was independently associated with CRCBSI (odds ratio $=1.142,95 \%$ confidence interval $=1.049-1.244$, $P=0.002)$. Solid tumors $(P=0.08)$, chronic hepatic insufficiency $(P=0.82)$, age $(P=0.16)$ and body weight $(P=0.58)$ were not associated with CRCBSI (Table 4).

\section{Antifungal treatment}

Twenty-eight CRCBSI patients $(28 / 29,96.6 \%)$ received antifungal treatment, without difference from the NCRCBSI group $(229 / 265,86.4 \% ; P>0.05)$ (Table 1$)$. The more frequently used drugs were fluconazole, followed by caspofungin and voriconazole. The course of antifungal therapy was similar between the two groups. More CRCBSI patients 
Table 1 Baseline characteristics of 294 patients with Candida bloodstream infection in the CHINA-SCAN study, according to CRCBSI and NCRCBSI

\begin{tabular}{|c|c|c|c|}
\hline \multirow[t]{2}{*}{ Variables } & \multirow{2}{*}{$\begin{array}{l}\text { CRCBSI } \\
N=29\end{array}$} & \multirow{2}{*}{$\begin{array}{l}\text { NCRCBSI } \\
N=265\end{array}$} & \multirow[t]{2}{*}{$P$-value } \\
\hline & & & \\
\hline Age (years), mean $\pm S D$ & $69.4 \pm 19.1$ & $60.7 \pm 20.2$ & $0.028^{*}$ \\
\hline Gender, n (\%) & & & 0.527 \\
\hline Male & $22(75.9)$ & $181(68.3)$ & \\
\hline Female & $7(24.1)$ & $84(31.7)$ & \\
\hline Body weight $(\mathrm{kg})$, mean \pm SD & $58.0 \pm 5.2$ & $63.2 \pm 11.0$ & $0.037^{*}$ \\
\hline \multicolumn{4}{|l|}{ Symptoms, n (\%) } \\
\hline Fever & $27(93.1)$ & $243(91.7)$ & 1.000 \\
\hline Shivers & $8(27.6)$ & $83(31.3)$ & 0.833 \\
\hline Confusion & $13(44.8)$ & $123(46.4)$ & 1.000 \\
\hline \multicolumn{4}{|l|}{ Concomitant disease, n (\%) } \\
\hline Type 1 or 2 diabetes & $7(24.1)$ & $59(22.3)$ & 0.833 \\
\hline Chronic cardiac dysfunction & $6(20.7)$ & $57(21.5)$ & 0.891 \\
\hline Solid tumor & $8(27.6)$ & $45(16.9)$ & 0.180 \\
\hline Chronic obstructive pulmonary disease & $4(13.8)$ & $31(11.7)$ & 0.762 \\
\hline Chronic renal insufficiency & $5(17.2)$ & $27(10.2)$ & 0.259 \\
\hline Chronic hepatic insufficiency & $4(13.8)$ & $12(4.5)$ & 0.060 \\
\hline Hematological malignancy & $0(0.0)$ & $3(1.2)$ & 1.000 \\
\hline \multicolumn{4}{|c|}{ Invasive procedures within 2 weeks prior to diagnosis, n (\%) } \\
\hline Hemodialysis & $2(6.9)$ & $15(5.7)$ & 0.259 \\
\hline Invasive mechanical ventilation & $24(82.7)$ & $204(77.0)$ & 0.666 \\
\hline Total parenteral nutrition & $14(48.3)$ & $115(43.4)$ & 0.695 \\
\hline Surgery & $11(37.9)$ & $102(38.5)$ & 1.000 \\
\hline Immunosuppression & $2(6.9)$ & $15(5.7)$ & 0.679 \\
\hline \multicolumn{4}{|l|}{ IIIness severity at ICU admission, mean \pm SD } \\
\hline APACHE II score & $28.5 \pm 7.6$ & $27.0 \pm 7.1$ & 0.286 \\
\hline SOFA score & $10.6 \pm 2.9$ & $11.2 \pm 3.5$ & 0.330 \\
\hline \multicolumn{4}{|l|}{ Illness severity at diagnosis, mean \pm SD } \\
\hline APACHE II score & $28.2 \pm 7.2$ & $27.0 \pm 7.0$ & 0.360 \\
\hline SOFA score & $9.8 \pm 3.3$ & $10.8 \pm 3.5$ & 0.147 \\
\hline Immune enhancement therapy, $\mathrm{n}(\%)^{\mathrm{a}}$ & $21(72.4)$ & $102(38.5)$ & $<0.001^{* *}$ \\
\hline Antibiotic use, n (\%) & & & 0.963 \\
\hline Monotherapy & $8(32.0)$ & 75 (35.9) & \\
\hline Two-drug combinations & $13(52.0)$ & $98(46.9)$ & \\
\hline Three-drug combinations & $4(16.0)$ & $35(16.7)$ & \\
\hline Antibiotic use period, mean \pm SD & $11.4 \pm 4.2$ & $10.6 \pm 6.5$ & 0.514 \\
\hline Antibiotic therapy >5 days, n (\%) & $25(86.2)$ & $209(78.9)$ & 0.469 \\
\hline Antifungal therapy, n (\%) & $28(96.6 \%)$ & $229(86.4 \%)$ & 0.118 \\
\hline Initial antifungal treatment, n (\%) & & & 0.977 \\
\hline Fluconazole & $11(39.3 \%)$ & $84(36.7 \%)$ & \\
\hline Caspofungin & $7(25.0 \%)$ & $54(23.6 \%)$ & \\
\hline Voriconazole & $4(14.3 \%)$ & $44(19.2 \%)$ & \\
\hline Micafungin & $3(10.7 \%)$ & $20(8.7 \%)$ & \\
\hline
\end{tabular}




\author{
Table 1 Baseline characteristics of 294 patients with Candida bloodstream infection in the CHINA-SCAN study, \\ according to CRCBSI and NCRCBSI (Continued)

\begin{tabular}{|c|c|c|c|}
\hline Itraconazole & $3(10.7 \%)$ & $18(7.9 \%)$ & \\
\hline Amphotericin B (liposomes or lipid dispersions) & 0 & $5(2.2 \%)$ & \\
\hline Two-drugs combination ${ }^{\mathrm{b}}$ & 0 & $4(1.7 \%)$ & \\
\hline Treatment duration, mean \pm SD & $19.0 \pm 13.3$ & $16.7 \pm 13.3$ & 0.338 \\
\hline Antifungal therapy >5 days, n (\%) & $8(27.6)$ & $70(26.4)$ & 1.000 \\
\hline Time between ICU admission and diagnosis of Candida infection (days), median (Q1,Q3) & $11.0(4.0,26.0)$ & $10.00(4.0,21.0)$ & 0.544 \\
\hline
\end{tabular}

received immune enhancement therapy (immunoglobulins and/or thymosin $\alpha 1)(72.4 \%$ vs. 38.5\%; $P<0.001)$ (Table 1). However, because it only was an observational indicator, the types and doses of immunoglobulins and thymosin $\alpha 1$ were not recorded.

\section{Treatment outcomes}

CRCBSI patients showed a non-significant higher mortality (44.8\% vs. 36.2\%; $P=0.419$ ). Trends toward longer ICU stay (median: 34 vs. 25 days; $P=0.095$ ) and hospitalization (median: 54 vs. 39 days; $P=0.096$ ) were also observed. CRCBSI patients were more likely to experience microbiological recovery compared with NCRCBSI patients $(67.9 \%$ vs. $50.0 \%$; $P<0.001$ ) (Table 5).

\section{Discussion}

To our knowledge, the China-SCAN study is the largest prospective study of invasive candidiasis in Chinese ICUs, and possibly from anywhere. In addition, it is also one of the first to describe Candida catheter-related bloodstream infections in China. The present study aimed to determine the risk factors for catheter-related candidemia in Chinese ICU. Our results showed that CRCBSI incidence in ICU was $0.03 \%$, accounting for $9.86 \%$ of all candidemia observed in ICU (29/294), mainly caused by Candida parapsilosis in CRCBSI patients (33.3\%). Univariate analyses showed that older age and lower body weight were associated with CRCBSI. Multivariate analysis showed that the SOFA score was independently associated with CRCBSI $(P=0.002)$. Catheter removal and immune enhancement therapy were more frequently used in CRCBSI than in NCRCBSI. Results of the present study provide clues for a better identification of CRCBSI patients.

Few studies reported large-scale epidemiological data on CRCBSI. In the present study, we reported a CRCBSI incidence in ICUs of 0.3/1000 patients, which was calculated based on the 96,060 ICU patients reported in the China-SCAN study. In the present study, some patients with NCRCBSI did not have blood sample in venous catheter; therefore, some of these patients might in reality be CRCBSI cases. In addition, some Candida-positive patients could have been excluded because of the strict inclusion criteria of the CHINA-SCAN study. Therefore, the real CRCBSI incidence may be higher.

In the present study, the mortality rate from CRCBSI was $44.8 \%$, which was not significantly different from that of NCRCBSI (36.2\%). These rates are in agreement with the published global mortality rates of 30-61.8\% in hospital-based candidemia studies from western countries [5-8].

The CRCBSI and NCRCBSI groups were compared in order to identify risk factors for CRCBSI. Results showed that the two groups were similar in disease, invasive procedures, disease severity score, and the use of antibiotics within the past two weeks. However, in univariate analyses, there were significant differences in age and body weight. These results suggest that risk factors for CRCBSI, other candidemias and invasive candidiasis were similar in most ICU patients, except for those with an older age and lower body weight.

CVC is the most common type of catheter causing CRCBSI [28]. Studies have shown that CVC placement rate in candidemia patients is $80-96.7 \%$ [21]. Consistent with these results, the CVC placement rates in patients with CRCBSI and those with NCRCBSI in the present study were above $80 \%$ ( $89.7 \%$ and $81.9 \%)$, and there was no significant different between the two groups. The CVC placement position and indwelling period were similar in both groups, indicating that the initial placement position of catheter and catheter indwelling time were not the cause of CRCBSI. The catheter removal rate was not different within 2 weeks before diagnosis between the two groups, but was significantly higher after diagnosis in the CRCBSI group (82.8\%) compared with the NCRCBSI group (60.0\%), in compliance with previous studies and guidelines $[18,29,30]$. A recent study suggested that any delay in catheter removal and initiation of antifungal therapy was associated with increased mortality in CRCBSI patients; however, catheter removal had no impact on mortality of 
Table 2 Indwelling and removal of catheters according to CRCBSI and NCRCBSI

\begin{tabular}{|c|c|c|c|c|}
\hline \multirow[t]{2}{*}{ Category } & & \multirow{2}{*}{$\begin{array}{l}\text { CRCBSI } \\
N=29\end{array}$} & \multirow{2}{*}{$\begin{array}{l}\text { NCRCBSI } \\
\mathrm{N}=265\end{array}$} & \multirow[t]{2}{*}{$P$-value } \\
\hline & & & & \\
\hline \multicolumn{2}{|l|}{ Indwelling central venous catheter, $\mathrm{n}(\%)^{\mathrm{a}}$} & & & 0.438 \\
\hline \multicolumn{2}{|l|}{ Yes } & $26(89.7)$ & $217(81.9)$ & \\
\hline \multicolumn{2}{|l|}{ No } & $3(10.3)$ & $48(18.1)$ & \\
\hline \multicolumn{5}{|l|}{ Catheter indwelling position, n (\%) } \\
\hline \multicolumn{2}{|l|}{ Jugular vein } & $12(41.4)$ & $107(40.4)$ & 1.000 \\
\hline \multicolumn{2}{|l|}{ Subclavian vein } & $13(44.8)$ & $104(39.2)$ & 0.556 \\
\hline \multicolumn{2}{|l|}{ Femoral vein } & $8(27.6)$ & $75(28.3)$ & 1.000 \\
\hline \multicolumn{5}{|l|}{ Catheter removal from each position, $\mathrm{n}(\%)^{\mathrm{b}}$} \\
\hline \multirow[t]{2}{*}{ Jugular vein } & A & $2(6.9)$ & $19(7.2)$ & 0.335 \\
\hline & B & $0(0)$ & $22(8.3)$ & \\
\hline \multirow[t]{2}{*}{ Subclavian vein } & A & $5(17.2)$ & $17(6.4)$ & 0.251 \\
\hline & $\mathrm{B}$ & $2(6.9)$ & $29(10.9)$ & \\
\hline \multirow[t]{2}{*}{ Femoral vein } & A & $2(6.9)$ & $14(5.3)$ & 0.960 \\
\hline & B & $2(6.9)$ & $20(7.5)$ & \\
\hline \multicolumn{5}{|c|}{ Period of catheter indwelling in the last time (days), median (min, max) } \\
\hline \multicolumn{2}{|l|}{ Jugular vein } & $10.00(4.0,13.0)$ & $8.00(-1.0,382.0)$ & 0.827 \\
\hline \multicolumn{2}{|l|}{ Subclavian vein } & $14.00(1.0,30.0)$ & $9.00(-337.0,63.0)$ & 0.150 \\
\hline \multicolumn{2}{|l|}{ Femoral vein } & $5.00(1.0,18.0)$ & $9.00(-58.0,369.0)$ & 0.161 \\
\hline \multicolumn{2}{|l|}{ Catheter removal after diagnosis, n (\%) } & $24(82.8)$ & $159(60.0)$ & $0.016^{*}$ \\
\hline
\end{tabular}

NCRCBSI patients [18]. On the other hand, some studies argued that CVC removal do not affect prognosis of candidemia [31]. The 2012 ESCMID guidelines also pointed out that catheter removal is necessary for candidemia, but that antifungal treatment can be used if catheter removal is impossible [32].

In the present study, multivariate analysis showed that the SOFA score was the only independent variable associated with CRCBSI. We explored a number of factors that have been shown to be associated with candidemia in previous studies, but we did not observe any association

Table 3 Candida strains according to CRCBSI and NCRCBSI

\begin{tabular}{llll}
\hline Strain & $\begin{array}{c}\text { CRCBSI } \\
\text { N= } \mathbf{2 1}\end{array}$ & $\begin{array}{l}\text { NCRCBSI } \\
\mathbf{N}=\mathbf{2 1 6}\end{array}$ & P-value \\
\hline Candida strains isolates, n (\%) & & & 0.352 \\
Candida albicans & $6(28.6)$ & $87(40.3)$ & \\
Candida parapsilosis & $7(33.3)$ & $48(22.2)$ & \\
Candida tropicalis & $2(9.5)$ & $37(17.1)$ & \\
Candida glabrata & $2(9.5)$ & $25(11.6)$ & \\
Others & $4(19.0)$ & $19(8.7)$ & \\
\hline
\end{tabular}

between these parameters and CRCBSI. The study by MacDonald et al. [19] showed that hyperalimentation was the only independent risk factor for candidemia in an ICU pediatric population. Another pediatric study showed that CVC, cancer, recent use of vancomycin, and use of agents against anaerobic bacteria were independent factors associated with candidemia [8]. A study showed that independent predictors of biofilm-forming candidemia were the use of CVC, the use of urinary catheters, parenteral alimentation, and diabetes [15]. Finally, a study showed that an inadequate antifungal therapy, infection with biofilm-forming Candida species, and APACHE III scores were associated with higher Candida-related mortality [16]. However, these studies did not differentiate between CRCBSI and NCRCBSI. In addition, the only risk factor for candidemia that was common to these studies was the use of CVC.

Because there is variability in the resistance of Candida strains to storage and transport, some samples could not be tested in the central laboratory, and the exact distribution of the different Candida species might have suffered from this bias. However, we observed that CRCBSI was mainly associated with Candida parapsilosis, while NCRCBSI was mainly associated with Candida albicans, although the 
Table 4 Multivariate logistic regression analysis for exposure to potential risk factors for CRCBSI in ICU patients

\begin{tabular}{|c|c|c|c|c|c|c|c|}
\hline Variables & Estimate & $\begin{array}{l}\text { Standard } \\
\text { error }\end{array}$ & $\begin{array}{l}\text { Wald } \\
\text { chi-square }\end{array}$ & $\begin{array}{l}\text { Odds ratio } \\
\text { estimate }\end{array}$ & $\begin{array}{l}\text { Lower } 95 \% \\
\text { confidence limit }\end{array}$ & $\begin{array}{l}\text { Upper } 95 \% \\
\text { confidence limit }\end{array}$ & $P$-value \\
\hline Solid tumors (yes vs. other Concomitant disease) & 0.344 & 0.1954 & 3.1008 & 1.990 & 0.925 & 4.279 & 0.0783 \\
\hline $\begin{array}{l}\text { Chronic hepatic insufficiency (yes vs. other } \\
\text { concomitant disease) }\end{array}$ & -0.1596 & 0.7118 & 0.0503 & 0.852 & 0.211 & 3.44 & 0.8226 \\
\hline Age & 0.0112 & 0.00808 & 1.9336 & 1.011 & 0.995 & 1.027 & 0.1644 \\
\hline SOFA score at diagnosis & 0.1329 & 0.0436 & 9.2834 & 1.142 & 1.049 & 1.244 & $0.0023^{* *}$ \\
\hline Body weight & -0.00817 & 0.0149 & 0.3021 & 0.992 & 0.963 & 1.021 & 0.5825 \\
\hline
\end{tabular}

distribution of strains between the two groups was not different. This observation was consistent with previous studies on candidemia [6,25,33]. Candida parapsilosis is more prone to cause CRCBSI, which may be related to its ease of growing in intravenous infusion of high-sugar-based nutrition, to its growing in CVC biofilm that can easily be spread by the hands of medical personnel, and to its long-term survival [33]. Using catheter removal and appropriate antifungal therapy, CRCBSI microbiological clearance rate was significantly higher than that of NCRCBSI $(67.9 \%$ vs. $50.0 \%)$, which was consistent with a previous study [18].

There was no difference in antifungal treatment between CRCBSI and NCRCBSI patients in respect to antifungal treatment. The most commonly used was fluconazole, followed by caspofungin and voriconazole. In the China-SCAN flora and sensitivity analysis, patients with non-albicans strains were more susceptible to require a therapy adjustment [10]. Considering the high proportion of Candida parapsilosis causing CRCBSI in the present study, we suggest to consider drugs with a higher efficacy against Candida parapsilosis in the treatment of CRCBSI.

Although there is no clear indicator of the presence of immunosuppression in the present study, ICU physicians rely on clinical experience to use immunotherapy. More CRCBSI patients received immune enhancement therapy $(72.4 \%$ vs. $38.5 \%)$, suggesting that ICU physicians are concerned about CRCBSI-related immune suppression. From the previously identified risk factors in patients with CRCBSI [8,15-19], patients with old age and low body weight might be more prone to develop immunosuppression. Although no immune enhancement therapy is clearly recognized to improve CRCBSI patients' prognosis, immunoglobulins and thymosin $\alpha 1$ were selected as immunotherapy since these drugs have a potential to improve prognosis in sepsis patients [34,35].

The China-SCAN study suffered from some limitations that also have an impact in the present study. Indeed, the lack of central validation for some samples could lead to an underestimation of the real CRCBSI incidence, as well as for the Candida strains causing CRCBSI. However, results based from the central laboratory clearly demonstrated that the proportion of Candida parapsilosis was higher in the CRCBSI group, while that of Candida albicans was higher in the NCRCBSI group. Differences in therapeutic strategies across the study centers may have contributed to biases in diagnosis, treatments and prognosis. Concerning the present study, the sample size of the CRCBSI group was small. Therefore, results need to be verified in further large-scale studies on CRCBSI.

\section{Conclusions}

In China, CRCBSI was more likely to occur in old patients with low body weight. SOFA score was independently associated with CRCBSI. Candida parapsilosis accounted for a high proportion of CRCBSI, but the difference from NCRCBSI was not significant.

Table 5 Treatment outcomes according to CRCBSI/NCRCBSI

\begin{tabular}{llll}
\hline Category & CRCBSI & NCRCBSI & $\mathbf{P}=\mathbf{2 6 5}$ \\
\hline Mortality, $\mathrm{n}(\%)$ & $\mathbf{n = 2 9}$ & $96(36.2)$ & 0.419 \\
ICU stay period (days), median (Q1,Q3) & $13(44.8)$ & $25.00(13.0,44.0)$ \\
Hospital stays (days), median (Q1,Q3) & $34.00(18.0,71.0)$ & $39.00(18.0,69.5)$ & 0.095 \\
Candida elimination, $n$ (\%) & $54.00(26.0,91.0)$ & $116(50.0)$ & 0.096 \\
Time from positive to negative blood culture (days), median (Q1,Q3) & $19(67.9)$ & $14.00(6.0,24.0)$ & $17.00(12.0,26.0)$ \\
\hline
\end{tabular}

${ }^{*} P<0.01$ 


\section{Additional file}

Additional file 1: Table S1. Candida strains by center.

\section{Competing interests}

Haibo Qiu is a speaker for Pfizer and MSD China, and has received research grants from Pfizer, MSD China and Xian-Janssen. The remaining authors have no conflicts of interest to disclose. The funders participated in the design of the study, but had no role in study management, monitoring data management, statistical analysis or development of this article. Authors accept direct responsibility for this paper.

\section{Authors' contributions}

$\mathrm{BH}, J \mathrm{~L}$ and $\mathrm{HQ}$ designed the study. $\mathrm{BH}, \mathrm{ZD}$ contributed to the manuscript development. ZD, YK, BZ, WC, BQ, QF and HQ were involved in patient recruitment and served as study investigators. All authors read and approved the final manuscript.

\section{Acknowledgements}

This study was supported by Merck Sharp \& Dohme China.

\section{Author details}

'Department of Intensive Care Unit, Zhongnan Hospital of Wuhan University, Wuhan, Hubei 430071, China. ${ }^{2}$ Department of Intensive Care Unit, West China Hospital, Sichuan University, Chengdu, China. ${ }^{3}$ Department of Intensive Care Unit, Shengjing Hospital, affiliated to China Medical University, Shenyang, China. ${ }^{4}$ Department of Intensive Care Unit, The 2nd Affiliated Hospital of Zhejiang University School of Medicine, Hangzhou, China. ${ }^{5}$ Department of Intensive Care Unit, Henan Provincial People's Hospital, Zhengzhou, China. ${ }^{6}$ The First Affiliated Hospital of Medical School of Zhejiang University, Hangzhou, China. ${ }^{7}$ Department of Intensive Care Unit, Nanjing Zhong-da Hospital, Southeast University School of Medicine, Nanjing, China.

Received: 19 May 2014 Accepted: 28 October 2014

Published online: 13 November 2014

\section{References}

1. Wisplinghoff $H$, Bischoff $T$, Tallent SM, Seifert $H$, Wenzel RP, Edmond MB: Nosocomial bloodstream infections in US hospitals: analysis of 24,179 cases from a prospective nationwide surveillance study. Clin Infect Dis 2004, 39:309-317.

2. Richards MJ, Edwards JR, Culver DH, Gaynes RP: Nosocomial infections in combined medical-surgical intensive care units in the United States. Infect Control Hosp Epidemiol 2000, 21:510-515.

3. Jarvis WR: Epidemiology of nosocomial fungal infections, with emphasis on Candida species. Clin Infect Dis 1995, 20:1526-1530.

4. Kett DH, Azoulay E, Echeverria PM, Vincent $J \mathrm{~L}$, Extended Prevalence of Infection in ICUSGol: Candida bloodstream infections in intensive care units: analysis of the extended prevalence of infection in intensive care unit study. Crit Care Med 2011, 39:665-670.

5. Bougnoux ME, Kac G, Aegerter P, d'Enfert C, Fagon JY, CandiRea Study G: Candidemia and candiduria in critically ill patients admitted to intensive care units in France: incidence, molecular diversity, management and outcome. Intensive Care Med 2008, 34:292-299.

6. Dimopoulos G, Ntziora F, Rachiotis G, Armaganidis A, Falagas ME: Candida albicans versus non-albicans intensive care unit-acquired bloodstream infections: differences in risk factors and outcome. Anesth Analg 2008, 106:523-529. table of contents.

7. Gudlaugsson O, Gillespie S, Lee K, Vande Berg J, Hu J, Messer S, Herwaldt L, Pfaller M, Diekema D: Attributable mortality of nosocomial candidemia, revisited. Clin Infect Dis 2003, 37:1172-1177.

8. Zaoutis TE, Argon J, Chu J, Berlin JA, Walsh TJ, Feudtner C: The epidemiology and attributable outcomes of candidemia in adults and children hospitalized in the United States: a propensity analysis. Clin Infect Dis 2005, 41:1232-1239.

9. Pappas PG, Kauffman CA, Andes D, Benjamin DK, Calandra TF, Edwards JE Jr, Filler SG, Fisher JF, Kullberg BJ, Ostrosky-Zeichner L, Reboli AC, Rex JH, Walsh TJ, Sobel JD, Infectious Diseases Society of America: Clinical practice guidelines for the management of candidiasis: 2009 update by the Infectious Diseases Society of America. Clin Infect Dis 2009, 48:503-535.
10. Guo F, Yang $Y$, Kang $Y$, Zang B, Cui W, Qin B, Qin $Y$, Fang Q, Qin T, Jiang D, Li W, Gu Q, Zhao H, Liu D, Guan X, Li J, Ma X, Yu K, Chan D, Yan J, Tang Y, Liu W, Li R, Qiu H, China-SCAN Team: Invasive candidiasis in intensive care units in China: a multicentre prospective observational study. J Antimicrob Chemother 2013, 68:1660-1668

11. Blot F, Nitenberg G, Chachaty E, Raynard B, Germann N, Antoun S, Laplanche A, Brun-Buisson C, Tancrede C: Diagnosis of catheter-related bacteraemia: a prospective comparison of the time to positivity of hub-blood versus peripheral-blood cultures. Lancet 1999, 354:1071-1077.

12. Almirante B, Rodríguez D, Park BJ, Cuenca-Estrella M, Planes AM, Almela M, Mensa J, Sanchez F, Ayats J, Gimenez M, Saballs P, Fridkin SK, Morgan J, Rodriguez-Tudela JL, Warnock DW, Pahissa A, Barcelona Candidemia Project Study Group: Epidemiology and predictors of mortality in cases of Candida bloodstream infection: results from population-based surveillance, barcelona, Spain, from 2002 to 2003. J Clin Microbiol 2005, 43:1829-1835.

13. Blumberg HM, Jarvis WR, Soucie JM, Edwards JE, Patterson JE, Pfaller MA Rangel-Frausto MS, Rinaldi MG, Saiman L, Wiblin RT, Wenzel RP, National Epidemiology of Mycoses Survey(NEMIS) Study Group: Risk factors for candidal bloodstream infections in surgical intensive care unit patients: the NEMIS prospective multicenter study. The National Epidemiology of Mycosis Survey. Clin Infect Dis 2001, 33:177-186.

14. Gonzalez CE, Venzon D, Lee S, Mueller BU, Pizzo PA, Walsh TJ: Risk factors for fungemia in children infected with human immunodeficiency virus: a case-control study. Clin Infect Dis 1996, 23:515-521.

15. Tumbarello M, Fiori B, Trecarichi EM, Posteraro P, Losito AR, De Luca A, Sanguinetti M, Fadda G, Cauda R, Posteraro B: Risk factors and outcomes of candidemia caused by biofilm-forming isolates in a tertiary care hospital. PLoS One 2012, 7:e33705.

16. Tumbarello M1, Posteraro B, Trecarichi EM, Fiori B, Rossi M, Porta R, de Gaetano Donati K, La Sorda M, Spanu T, Fadda G, Cauda R, Sanguinetti M: Biofilm production by Candida species and inadequate antifungal therapy as predictors of mortality for patients with candidemia. J Clin Microbiol 2007, 45:1843-1850.

17. Dotis J, Prasad PA, Zaoutis T, Roilides E: Epidemiology, risk factors and outcome of Candida parapsilosis bloodstream infection in children. Pediatr Infect Dis J 2012, 31:557-560.

18. Garnacho-Montero J, Diaz-Martin A, Garcia-Cabrera E, Ruiz Perez de Pipaon M, Hernandez-Caballero C, Lepe-Jimenez JA: Impact on hospital mortality of catheter removal and adequate antifungal therapy in Candida spp. bloodstream infections. J Antimicrob Chemother 2013, 68:206-213.

19. MacDonald L, Baker C, Chenoweth C: Risk factors for candidemia in a children's hospital. Clin Infect Dis 1998, 26:642-645

20. Mean M, Marchetti $O$, Calandra T: Bench-to-bedside review: Candida infections in the intensive care unit. Crit Care 2008, 12:204

21. Montagna MT, Caggiano G, Lovero G, De Giglio O, Coretti C, Cuna T, latta R, Giglio M, Dalfino L, Bruno F, Puntillo F: Epidemiology of invasive fungal infections in the intensive care unit: results of a multicenter Italian survey (AURORA Project). Infection 2013, 41:645-653.

22. Pfaller MA, Diekema DJ: Epidemiology of invasive candidiasis: a persistent public health problem. Clin Microbiol Rev 2007, 20:133-163.

23. Horasan ES, Ersoz G, Goksu M, Otag F, Kurt AO, Karacorlu S, Kaya A: Increase in Candida parapsilosis fungemia in critical care units: a 6-years study. Mycopathologia 2010, 170:263-268.

24. Ben-Ami R, Weinberger M, Orni-Wasserlauff $R$, Schwartz D, Itzhaki A, Lazarovitch T, Bash E, Aharoni Y, Moroz I, Giladi M: Time to blood culture positivity as a marker for catheter-related candidemia. J Clin Microbiol 2008, 46:2222-2226.

25. Liu W, Tan J, Sun J, Xu Z, Li M, Yang Q, Shao H, Zhang L, Liu W, Wan Z, Cui W, Zang B, Jiang D, Fang Q, Qin B, Qin T, Li W, Guo F, Liu D, Guan X, Yu K, Qiu H, Li R, China-SCAN team: Invasive candidiasis in intensive care units in China: in vitro antifungal susceptibility in the China-SCAN study. J Antimicrob Chemother 2014, 69:162-167.

26. Knaus WA, Draper EA, Wagner DP, Zimmerman JE: APACHE II: a severity of disease classification system. Crit Care Med 1985, 13:818-829.

27. Vincent JL, Moreno R, Takala J, Willatts S, De Mendonca A, Bruining H, Reinhart CK, Suter PM, Thijs LG: The SOFA (Sepsis-related Organ Failure Assessment) score to describe organ dysfunction/failure. On behalf of the Working Group on Sepsis-Related Problems of the European Society of Intensive Care Medicine. Intensive Care Med 1996, 22:707-710.

28. Glockner A, Karthaus M: Current aspects of invasive candidiasis and aspergillosis in adult intensive care patients. Mycoses 2011, 54:420-433. 
29. Mermel LA, Allon M, Bouza E, Craven DE, Flynn P, O'Grady NP, Raad II, Rijnders BJ, Sherertz RJ, Warren DK: Clinical practice guidelines for the diagnosis and management of intravascular catheter-related infection: 2009 Update by the Infectious Diseases Society of America. Clin Infect Dis 2009, 49:1-45.

30. Andes DR, Safdar N, Baddley JW, Playford G, Reboli AC, Rex JH, Sobel JD, Pappas PG, Kullberg BJ, Mycoses Study G: Impact of treatment strategy on outcomes in patients with candidemia and other forms of invasive candidiasis: a patient-level quantitative review of randomized trials. Clin Infect Dis 2012, 54:1110-1122.

31. Nucci M, Anaissie E, Betts RF, Dupont BF, Wu C, Buell DN, Kovanda L, Lortholary O: Early removal of central venous catheter in patients with candidemia does not improve outcome: analysis of 842 patients from 2 randomized clinical trials. Clin Infect Dis 2010, 51:295-303.

32. Cornely OA, Bassetti M, Calandra T, Garbino J, Kullberg BJ, Lortholary O, Meersseman W, Akova M, Arendrup MC, Arikan-Akdagli S, Bille J, Castagnola E, Cuenca-Estrella M, Donnelly JP, Groll AH, Herbrecht R, Hope WW, Jensen HE, Lass-Flörl C, Petrikkos G, Richardson MD, Roilides E, Verweij PE, Viscoli C, Ullmann AJ, ESCMID Fungal Infection Study Group: ESCMID* guideline for the diagnosis and management of Candida diseases 2012: non-neutropenic adult patients. Clin Microbiol Infect 2012, 18(Suppl 7):19-37.

33. Trofa D, Gacser A, Nosanchuk JD: Candida parapsilosis, an emerging fungal pathogen. Clin Microbiol Rev 2008, 21:606-625.

34. Kreymann KG, de Heer G, Nierhaus A, Kluge S: Use of polyclona immunoglobulins as adjunctive therapy for sepsis or septic shock. Crit Care Med 2007, 35:2677-2685.

35. Li Y, Chen H, Li X, Zhou W, He M, Chiriva-Internati M, Wachtel MS, Frezza EE: A new immunomodulatory therapy for severe sepsis: Ulinastatin Plus Thymosin \{alpha\} 1. J Intensive Care Med 2009, 24:47-53.

doi:10.1186/s12879-014-0594-0

Cite this article as: Hu et al: Catheter-related Candida bloodstream infection in intensive care unit patients: a subgroup analysis of the China-SCAN study. BMC Infectious Diseases 2014 14:594.

\section{Submit your next manuscript to BioMed Central and take full advantage of:}

- Convenient online submission

- Thorough peer review

- No space constraints or color figure charges

- Immediate publication on acceptance

- Inclusion in PubMed, CAS, Scopus and Google Scholar

- Research which is freely available for redistribution 